\title{
Generic Pharmaceuticals: Is Pharmacovigilance Required?
}

\section{Suprita A Tawde}

Akorn Pharmaceuticals Inc., USA

"Corresponding author: Suprita A Tawde, Akorn Pharmaceuticals Inc., USA, Tel: 847-990-1721; E-mail: Suprita.Tawde@akorn.com

Received date: November 16, 2014, Accepted date: November 18, 2014, Published date: November 25, 2014

Copyright: @ 2014 Tawde SA. This is an open-access article distributed under the terms of the Creative Commons Attribution License, which permits unrestricted use, distribution, and reproduction in any medium, provided the original author and source are credited.

\section{Editorial}

The generic pharmaceuticals are the largest contributor of drugs in the market and are in demand due to cost-effectiveness and low insurance premium. As the generic drugs are approved by FDA and are allowed to be marketed only when the brand drug has been there in the market for years, it has well established safety profile. Therefore, unforeseen adverse events are very unlikely for such well known drugs. With numerous generic drugs entering in the pharmaceuticals market, the need of pharmacovigilance has become crucial for new as well as well-established drugs. Although generic medications appear to be same as brand drugs, there can be differences based upon different manufacturers. The question of requirement of pharmacovigilance for generics arises when there is plethora of information available for brand drug regarding safety and adverse events.

Generic drugs are same in dosage form, safety, strength, route of administration, quality, performance characteristics and intended use as indicated for reference listed drugs (RLD) or brand drugs. Though all the components of generic formulations are similar to brand drug there can be some differences based upon source of excipients. Excipients are the substances added to the formulation to render the drug in a compatible form for administration or for stability of drug over shelf life. These excipients are inactive substances with no effect on drug action as such. However, there have been reports of instability and adverse events caused due to excipients $[1,2]$. On the other hand, biosimilars are expected to have no clinical differences when compared to brand drugs. However, biosimilars may have minor structural differences which may attribute to adverse events related to immunogenicity.

Another factor which can contribute to differences in generics from brand drugs is incompatible container closure system. Recently some injectable drugs faced recalls from market due to issues found with the product. One of major issues found with injectables is the presence of foreign particulate matter in product. Some examples of such recalls reported by FDA are Cubicin (Daptomycin for Injection) manufactured by Cubist Pharmaceuticals, Sodium Chloride Injection by Baxter, Lactated Ringers and 5\% Dextrose Injection by Hospira Inc [3]. Moreover, glass de-lamination can occur due container/ closure system selected. Therefore, a stringent pharmacovigilance study is required to ensure that any issues related to excipients or container closure systems are adequately addressed. Moreover, the approval process for generic drugs does not involve complete clinical trials as in case of brand drugs. The lack of clinical trials makes the need of pharmacovigilance essential for generic drugs. In case of biosimilars, clinical trials required for approval may not detect rare adverse events or those occurring after prolonged use, and the incidence of such events may differ between a biosimilar and its brand drug [4,5].

In addition, a critical issue faced by generic drugs, especially biosimilars, is the adverse event reporting system utilized for branded drugs and their generic versions. Biosimilars are a new class of drugs entering the US market in early 2015 . These are very similar to the brand drug; however there can be differences due to their structural complexity and manufacturing process variations among companies. The safety surveillance systems followed in the US are currently through insurance claims database, pharmacy benefits or medical benefits. Another resource of such events is spontaneous voluntary reports submitted to FDA (FAERS- FDA Adverse Events Reporting System) by health professionals and consumers [6]. The manufacturers are required by law to report adverse event to FDA in case they receive such information. However, there are no means which can accurately distinguish product specific safety signals for generics manufactured by different pharmaceutical companies. More than $90 \%$ of FAERS are reported without lot numbers and National Drug Code (NDC) numbers are not included in such reporting systems. It has been found that the adverse events reporting for brand drugs remained same even after entry of generic drugs in the market. In most of the cases, patients are switched from brand drugs to generics once generic drugs are available in order to lower insurance premiums. However, the adverse events are attributed to the brand drug even after the switch to generic drugs [7]. Therefore, a better pharmacovigilance system is required to detect specific adverse events with respect to the responsible product. The need for biologic identification needs further evaluation by the possibility of biosimilar substitution without prescriber intervention. In contrast, generic small-molecule drugs are identical to their reference product and therefore they have the same nonproprietary name. There have been discussion to evaluate the suggestions to improve the current pharmacovigilance system followed based on distinguishable brand names, involvement of NDCs, and lot numbers in reports [5,8].

In conclusion, pharmacovigilance will continue to provide better safety aspects for both brand as well as generic drugs. This safety surveillance system needs some modifications to identify productspecific adverse events especially in case of biosimilars and to distinguish between products, avoid medication errors, and facilitate effective pharmacovigilance.

\section{Reference}

1. Why pharmaceutical companies Need post marketing Pharmacovigilance for well- established drugs.

2. Corbet B (2007) Manual of Drug Safety and Pharmacovigilance. (1stedn), Jones and Bartlett, Massachusetts.

3. Recalls, Market Withdrawals, \& Safety Alerts. U S Food and Drug Administration.

4. Jeetu G, Anusha G (2010) Pharmacovigilance: A Worldwide Master Key for Drug Safety Monitoring. J Young Pharm 2: 315-320.

5. Casadevall N, Felix T, Strober BE, Warnock DG (2014) Similar names for similar biologics. BioDrugs 28: 439-444. 
Citation: Tawde SA (2014) Generic

Pharmaceuticals: Is Pharmacovigilance

Required?. J Pharmacovigil

2: e124. doi: $10.4172 / 2329-6887.1000 \mathrm{e} 124$

Page 2 of 2

6. FDA Adverse Event Reporting System (FAERS). U S Food and Drug Administration.

7. Emily Alexander (2014) Impact of Non- Proprietary Names on Pharmacovigilance.

8. Miller S (2013) Is it necessary to depart from international naming conventions for biosimilars in the US to ensure the safety of biologic and biosimilar therapeutics?: A response to 'It's all about the name: What is the imperative of adopting unique names for biologic and biosimilar therapeutics?.' Food and Drug Policy Forum 3: 1-24. 